\title{
Catatonia: a propósito de un caso.
}

Catatonia: a case study.

\author{
Nora Olazabal Eizaguirre a, Sonia Bustamante Madariaga b, Goizalde Solano Iturri c, Reda Rah- \\ mani $^{\text {d, Leire Erkoreka Gonzalez }}{ }^{\mathrm{e}}$, Miguel Ángel Gonzalez-Torres ${ }^{\mathrm{f}}$.
}

\begin{abstract}
${ }^{a}$ Servicio de Psiquiatría. Hospital de Basurto. Osakidetza, Bilbao. ${ }^{b}$ Servicio de Psiquiatría. Hospital de Basurto. Osakidetza, Bilbao. 'Servicio de Medicina Interna. Hospital de Basurto. Osakidetza, Bilbao. ${ }^{d}$ Servi-

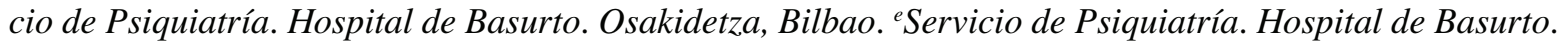

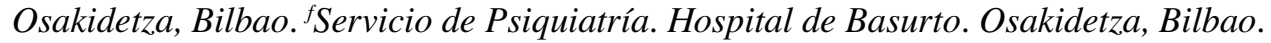

Correspondencia: Nora Olazabal Eizaguirre (nora.olazabaleizaguirre@osakidetza.net)

Recibido: 4/06/2010; aceptado con modificaciones: 3/09/2010

RESUMEN: Presentamos un cuadro clínico de mutismo, acinesia y estupor con fiebre y retención urinaria en una mujer de 65 años como ejemplo de catatonia. La sintomatología catatónica se ha establecido como un síndrome común a múltiples etiologías tanto médicas como psiquiátricas. Además se han descrito factores precipitantes de tipo farmacológico, tóxico y orgánico para esta entidad. Por tanto, es necesaria una aproximación multidisciplinar a este tipo de cuadros para afinar el diagnóstico etiológico.

Varios autores apuntan a un infradiagnóstico de este síndrome. Durante la evaluación, diagnóstico y tratamiento de esta paciente, hallamos la necesidad de criterios diagnósticos claros y actualizados y de algoritmos de tratamiento basados en evidencias. Las benzodiazepinas y la terapia electroconvulsiva suponen el tratamiento de primera línea, junto con las medidas de soporte y la prevención de complicaciones. Se han publicado otras estrategias no protocolizadas de tratamiento alternativas en casos refractarios.

PALABRAS CLAVE: catatonia, manía, síndrome neuroléptico maligno, lorazepam, terapia electroconvulsiva.
ABSTRACT: We present a clinical picture of mutism, akinesia and stupor with fever and urinary retention in a 65-year-old woman, as an example of catatonia. The catatonic symptomatology has been established as a syndrome which can have multiple etiologies, both medical and psychiatric. Beside that, pharmacological, toxic and organic precipitant factors have been described. Therefore a multidisciplinary approach is required to make more precise the etiological diagnosis.

Many authors point out that this syndrome is underdiagnosed. During the assessment, diagnosis and treatment of this patient, we found that there is lack of clear and updated diagnostic criteria, as well as evidence-based treatment algorithms. Benzodiazepines and ECT are first line treatments, along with supportive care and prevention of complications. Other non-protocolized strategies have been published as an alternative in refractory cases.

KEY WORDS: catatonia, mania, neuroleptic malignant syndrome, lorazepam, electroconvulsive therapy.

\section{Introducción}

Desde las primeras descripciones del síndrome catatónico por Kahlbaum (1) el concepto de catatonia ha sufrido una gran evolución. Si bien Kraepelin y Bleuler $(2,3)$ lo relacionaron principalmente con la dementia praecox y la esquizofrenia respectivamente, posteriormente se ha evidenciado que su incidencia es mayor en los trastornos afectivos y sobre todo en la manía. En la primera mitad del siglo XX el diagnóstico de catatonia disminuyó notablemente, hecho que se atribuyó al uso ex- 
Tabla 1

Criterios diagnósticos del DSM IV y criterios propuestos por Taylor y Fink.

\begin{tabular}{|c|c|}
\hline Criterios diagnósticos DSM- IV & Criterios diagnósticos Taylor y Fink, 2003 \\
\hline $\begin{array}{l}\text { Al menos dos de los siguientes síntomas: } \\
\text { 1. Inmovilidad motora manifestada por catalepsia } \\
\text { (incluida la flexibilidad cérea) o estupor } \\
\text { 2. Actividad motora excesiva (que aparentemente } \\
\text { carece de propósito y no está influida por estímulos } \\
\text { externos) } \\
\text { 3. Negativismo extremo (resistencia aparentemente } \\
\text { inmotivada a todas las órdenes o mantenimiento de } \\
\text { una postura rígida en contra de los intentos de ser } \\
\text { movido) o mutismo } \\
\text { 4. Peculiaridades del movimiento voluntario } \\
\text { manifestadas por la adopción de posturas extrañas } \\
\text { (adopción voluntaria de posturas raras o inapropiadas), } \\
\text { movimientos estereotipados, manierismos marcados } \\
\text { o muecas llamativas } \\
\text { 5. Ecolalia o ecopraxia }\end{array}$ & $\begin{array}{l}\text { Un criterio de tipo A o dos criterios de tipo B con } \\
\text { duración de una hora o más o que se reproducen en } \\
\text { dos o más ocasiones: } \\
\text { A. Inmovilidad, mutismo o estupor de más de una } \\
\text { hora de duración, asociado al menos a uno de los } \\
\text { siguientes: catalepsia, obediencia automática, } \\
\text { mantenimiento de posturas, observadas o referidas } \\
\text { en dos o más ocasiones. } \\
\text { B. En ausencia de inmovilidad, mutismo o estupor, } \\
\text { dos de los siguientes: estereotipias, ecofenómenos, } \\
\text { catalepsia, obediencia automática, mantenimiento de } \\
\text { posturas, negativismo, ambitendencia. }\end{array}$ \\
\hline
\end{tabular}

tendido de los antipsicóticos y al proceso de desinstitucionalización (4). Sin embargo, en 2003 Taylor y Fink publican un libro monográfico sobre la catatonia (5), donde se revisan exhaustivamente los criterios diagnósticos, alertando sobre el infradiagnóstico y enfatizando sobre la necesidad de escalas que amplíen los ítems diagnósticos del DSM-IV para obtener una mayor sensibilidad en el diagnóstico. Ese trabajo y otros posteriores ponen énfasis en el tratamiento sindrómico (independiente de la etiología) de la catatonia $(4,5)$, definiéndolo como un síndrome de disregulación motora (5). La bibliografía actual aconseja también la utilización de escalas específicas para diagnosticar y valorar la evolución $(6,7)$.

Además de las enfermedades psiquiátricas clásicas (manía, depresión melancólica, psicosis), son causa del síndrome catatónico múltiples condiciones médicas generales (infecciones, síndromes paraneoplásicos, patología endocrinológica, etc.) (8-10). Algunos fármacos pueden actuar como precipitantes de síndromes catatónicos (4), y se ha corroborado la estrecha relación entre la catatonia, el síndrome neuroléptico maligno y el síndrome tóxico serotoninérgico (11-14). La disminución de la prevalencia que desde los textos clásicos hasta hoy observamos se ha justificado por la menor monitorización actual de los signos motores en pacientes psiquiátricos, la proliferación del uso de antipsicóticos y el mayor esfuerzo en rehabilitar a los pacientes psiquiátricos graves. Sin embargo, esta disminución parece parcialmente ilusoria, pues aun con tratamientos modernos la catatonia continúa existiendo y probablemente esté infradiagnosticada $(4,5,15-17)$. 
NOTAS CLÍNICAS

Se establece la prevalencia de cuadros catatónicos en pacientes psiquiátricos ingresados en $9-15 \%(4,5)$. La manía es la principal causa de catatonia de origen psiquiátrico. Algunas series de casos establecen que el $15 \%$ de los pacientes ingresados por manía presentan signos de catatonia y que el $66 \%$ de los pacientes catatónicos ingresados cumplen criterios de trastorno afectivo $(5,18)$.

Presentamos a continuación el ejemplo de un caso clínico, con el objetivo de ilustrar la presentación, el enfoque diagnóstico y el tratamiento de la catatonía en la actualidad.

\section{Caso clínico}

Mujer de 65 años, con diagnóstico previo de trastorno afectivo bipolar tipo II tratada con litio y olanzapina que acude al Servicio de Urgencias por alteraciones de conducta de cuatro días de evolución. La paciente presenta antecedentes de larga data con varios ingresos en servicios de psiquiatría. Vive sola y es autónoma para las actividades de la vida diaria aunque recibe ayuda domiciliaria. La familia refiere clínica inicial de oscilaciones del estado de ánimo y vestimenta extravagante que posteriormente evoluciona a disartria, dificultad en la marcha y desorientación temporo-espacial. En el estudio realizado en el Servicio de Urgencias se observa hipertensión arterial $(174 / 110 \mathrm{mmHg})$, hipopotasemia $(3,09 \mathrm{mEq} / \mathrm{l})$, fiebre $\left(38,4^{\circ} \mathrm{C}\right)$ y distensión abdominal que se atribuyó a retención urinaria, extrayéndose 2 litros de orina tras sondaje vesical. La litemia, el ECG, la CK y la TAC craneal son normales, también la radiografía simple de abdomen y tórax. Se realiza punción lumbar con recuento celular normal. Se ingresa a la paciente en Servicio de Medicina Interna con impresión diagnóstica de síndrome confusional sin etiología filiada. Se trata con antipiresis, antibioterapia de amplio espectro y profilaxis de trombosis venosa con heparina. Se amplia el estudio con nueva punción lumbar, RM de cráneo, TAC torácico y abdómino-pélvico, hemocultivos y urocultivos que no presentan alteraciones que justifiquen el cuadro. El cuadro evoluciona en dos días a mutismo, acinesia, rigidez, temblor distal y disminución del parpadeo. Persiste la fiebre y la hipertensión arterial (HTA), con hipernatremia $(150 \mathrm{mEq} / 1)$. Las alteraciones electrolíticas se interpretan en el contexto de diaforesis y nula ingesta de líquido y alimento. Tras deterioro del nivel de conciencia se valora ingreso en Unidad de Reanimación y colocación de sonda nasogástrica para nutrición de la paciente. Se solicita valoración por Psiquiatría y se pauta terapia electroconvulsiva (TEC) urgente para el día siguiente con impresión diagnóstica de catatonia maligna. Dos días después de la primera sesión se constata que la paciente mantiene discurso espontáneo coherente y se alimenta. Se administran 2 sesiones más de TEC y se reintroduce el litio. La fiebre, la diaforesis, la HTA, 
las alteraciones electrolíticas y la desorientación han remitido. El cultivo para Virus Herpes Simple fue negativo, descartándose encefalitis por esta causa. Se procede al alta en 30 días con diagnóstico de TAB tipo II y catatonia maligna. Al alta la paciente se encontraba eutímica.

\section{Discusión}

\section{Diagnóstico sindrómico}

Los criterios diagnósticos del DSM-IV están asociados principalmente a dos etiologías: la esquizofrenia catatónica (F20.2) y la catatonia secundaria a enfermedad médica general o efecto adverso de medicación (F06.1). Para los diagnósticos de depresión y la manía la sintomatología catatónica se contempla como especificador (19). Varios artículos critican estos criterios por considerarlos limitados y favorecer el infradiagnóstico $(4,5,20)$. Taylor y Fink recomiendan el uso de unos criterios diagnósticos menos estrictos, siendo suficiente con un solo ítem de tipo A o con dos ítems del tipo B (Tabla 1) (5). Taylor y Fink van más allá y proponen que la catatonia sea una categoría diagnóstica independiente con especificadores que indiquen la causa: psiquiátrica, médica, tóxica (5). Se han elaborado algunas escalas (Rosebush, BFCR, MRS) cuya utilidad ha sido demostrada pero su aplicación en la práctica clínica no está extendida $(6,7)$.

Aunque clásicamente se haya aplicado el término de catatonia a estados de inhibición motriz, las presentaciones de este síndrome son variadas (16), pudiéndose alternar estados de inhibición y agitación en el mismo paciente. La catatonia inhibida, también llamada catatonia benigna o síndrome de Kahlbaum, es la presentación más frecuente en la clínica. El paciente responde muy pobremente a estímulos y puede llegar hasta el estado de estupor. Predominan los signos de acinesia y mutismo. El estado de conciencia, sin embargo, está conservado. Es necesario diferenciar esta entidad de depresiones mayores con inhibición psicomotora importante que no cursan con signos de catatonia. Estos pacientes pueden presentar agitación psicomotriz episódica. La catatonia agitada, se presenta con agitación motora excesiva, sin objetivo y lenguaje desorganizado. Predomina el estado de hipercinesia y puede acompañarse de desorientación, confusión y confabulación. Es importante diferenciar esta entidad de los estados de agitación en pacientes maníacos, ya que en el tratamiento de la catatonia agitada los antipsicóticos estarían contraindicados. La catatonia maligna se caracteriza por ser de inicio agudo y gran repercusión orgánica. Se acompaña frecuentemente de disautonomía, alteraciones en las pruebas de laboratorio y signos extrapiramidales en la exploración neurológica (5). Puede acompañarse de alteración del nivel de conciencia llegando hasta el coma, y si no se trata correctamente el pronóstico es sombrío (hasta 50\% de mortalidad). Este cuadro puede deberse a cuadros psiquiátricos, enfermedades médicas y a otras 
NOTAS CLÍNICAS

dos entidades de origen farmacológico: el síndrome neuroléptico maligno (SNM) y el síndrome serotoninérgico (SS). Estas entidades comparten fisiopatología, se consideran entidades nosológicas similares y se incluyen en el concepto de catatonia maligna (1114). La clínica del síndrome serotoninérgico puede incluir además, sintomatología del tracto gastrointestinal (sialorrea, dolor abdominal, vómitos, diarrea) (14).

De manera variable pero más frecuentemente asociados a la forma maligna (incluidos en el SNM y el SS), la presentación clínica se acompaña de disautonomía, alteraciones en las pruebas de laboratorio y signos extrapiramidales $(4,5)$. Los signos de disrregulación autonómica pueden cursar con hipertensión arterial, alteraciones en la frecuencia cardíaca, inestabilidad hemodinámica, diaforesis, etc. Los signos extrapiramidales son más frecuentes en la catatonia maligna, (incluídos el SNM y el $\mathrm{SS}$ ): rigidez muscular, temblor de reposo y rigidez en rueda dentada.

El aumento de CK es un hallazgo característico que puede ser útil en la confirmación de la sospecha de catatonia y hace necesaria la correcta hidratación del paciente catatónico. Al igual que el aumento de CK, las alteraciones hidroelectrolíticas actúan como causa y consecuencia. Está descrito que los estados de deshidratación actúan como precipitantes del síndrome (4). Los estados de ayuno, pobre ingesta de líquidos y diaforesis que pueden presentar estos pacientes pueden provocar estados de hipernatremia y aumento de CK.

De igual manera que en el delirium, se ha descrito, en algunos casos, actividad eléctrica cerebral enlentecida generalizada en pacientes con síndrome catatónico (5), que sin ser patognomónico, puede orientar en el diagnóstico.

En la literatura anglosajona se ha denominado test del lorazepam a la disminución de la inhibición motora y de la rigidez y mejoría transitoria del estado de estupor que presentan los pacientes catatónicos tras la administración de benzodiacepinas (BZD). Se considera que este ensayo de tratamiento puede ayudar al diagnóstico diferencial, ya que todas las formas de catatonia deberían responder favorablemente $(4,5,21,22)$.

\section{Diagnóstico etiológico}

La tabla 2 enumera las principales etiologías médicas, psiquiátricas y toxicológicas a las que se puede deber el síndrome catatónico. El estado clínico del paciente habitualmente dificulta la correcta anamnesis y el síndrome puede deberse a varias causas que actúan concomitantemente.

La historia psiquiátrica del paciente es de gran utilidad para establecer una hipótesis etiológica del cuadro. Además se debe estudiar con detenimiento y ayuda de acompañantes la clínica referida de los días previos al cuadro: desorganización conductual, alteraciones en el estado de ánimo y ritmos sueño-vigilia, verbalización de ideación delirante y alteraciones sensoroperceptivas.

Es imprescindible realizar una historia farmacológica exhaustiva que incluya tratamiento actual y tratamientos previos. De especial importancia son la toma y el 
Tabla 2

Etiologías más frecuentes del síndrome catatónico

\begin{tabular}{|l|l|}
\hline Trastornos psiquiátricos & Trastorno afectivo bipolar (episodios maníacos o mixtos) \\
& Depresión mayor \\
& Esquizofrenia paranoide \\
& Esquizofrenia catatónica \\
\hline Trastornos neurológicos & Epilepsia \\
& ACV \\
& Esclerosis múltiple \\
& Encefalitis \\
\hline Trastornos médicos & Patología endocrina \\
& Infecciones \\
& Alteraciones electrolíticas \\
& Síndrome paraneoplásico \\
\hline Fármacos y tóxicos & Deprivación: BZD, l-dopa, gabapentina \\
& Sobredosis: LSD, cocaína, anfetamina, disulfiram, levetiracetam \\
\hline
\end{tabular}

aumento de dosis en el caso de neurolépticos, tratamiento anticolinérgico, litio y disulfiram, también la sospecha de uso de tóxicos ilegales y la retirada o disminución de dosis de BZD o levodopa $(4,5)$. Se puede hallar bibliografía que relaciona todo tipo de fármacos con cuadros catatónicos: risperidona de liberación prolongada, anticonvulsivantes y antibióticos entre otros $(4,8,10,15)$.

El estudio orgánico del paciente debe ser llevado a cabo por especialistas que mediante exploración física y pruebas complementarias despisten un proceso orgánico (metabolopatía, masa intracraneal, crisis comiciales, enfermedad paraneoplásica, etc.). La exploración neurológica exhaustiva, las pruebas analíticas básicas y la monitorización de constantes son imprescindibles, así como los hemocultivos y los urocultivos en caso de que el paciente presente fiebre. El abordaje de estos pacientes debe ser eminentemente multidisciplinar y es aconsejable realizar pruebas de imagen cerebrales (TAC, $\mathrm{RM}$ ) que descarten un proceso sangrante o isquémico predominantemente en zona prefrontal, punción lumbar (PL) que descarte inflamación encefálica o de meninges, así como electroencefalograma (EEG) que descarte actividad epileptógena (5).

\section{Tratamiento}

El tratamiento del síndrome catatónico debe realizarse en régimen de hospitalización. Los cuidados básicos se basan en mantener el estado de nutrición e hidratación del paciente, movilizarlo para evitar úlceras, realizar cuidados en piel así como asegurar la seguridad del paciente (21). En pacientes con catatonia agitada puede ser necesaria la contención mecánica y la farmacológica, siendo las BZD fármacos de primera elección (4). Se debe evitar la iatrogenia por administración de fármacos que puedan empeorar el cuadro catatónico, principalmente los antipsicóticos de primera generación $(4,5)$.

El tratamiento inicial se realiza con BZD a dosis de 1-2 mg de lorazepam o equivalentes $(21,22)$. La vía de administración será intravenosa o intramuscular si 
el paciente no tolera la vía oral. Las BZD con más bibliografía disponible son el lorazepam (no disponible por vía parenteral en España) y el diazepam. Se recomienda la monitorización de los signos catatónicos una hora después de la administración, ya que se ha descrito que la mejoría de la clínica puede predecir la respuesta al tratamiento con BZD $(4,5,21,22)$. La dosis puede aumentarse hasta $24 \mathrm{mg} /$ día de lorazepam y debe mantenerse durante 6 días (4). Si el paciente no responde a BZD se aconseja el uso de TEC biparietal $(5,23,24)$. Si el estado orgánico del paciente estuviese comprometido (inestabilidad hemodinámica, disautonomía, hipertermia, otros signos de catatonia maligna) se recomienda utilizar TEC antes de los 6 días (5). Una vez obtenida respuesta los principales autores recomiendan mantener el tratamiento durante 6 meses (lorazepam o TEC de mantenimiento) (4).

Se ha postulado que la combinación de lorazepam y TEC podría tener una acción sinérgica (24). En ausencia de respuesta, o en caso de contraindicación de estos tratamientos, se han ensayado numerosos fármacos como alternativa (memantina, amantadina, bromocriptina, carbamazepina, topiramato, clozapina, valproato, olanzapina, litio), tanto en monoterapia como en combinación con lorazepam (21). Sin embargo existe una contradicción todavía no aclarada en estos tratamientos, ya que se ha descrito que algunos pueden actuar como precipitantes de cuadros de catatonia maligna o síndrome neuroléptico maligno.

Figura 1

Algoritmo de tratamiento

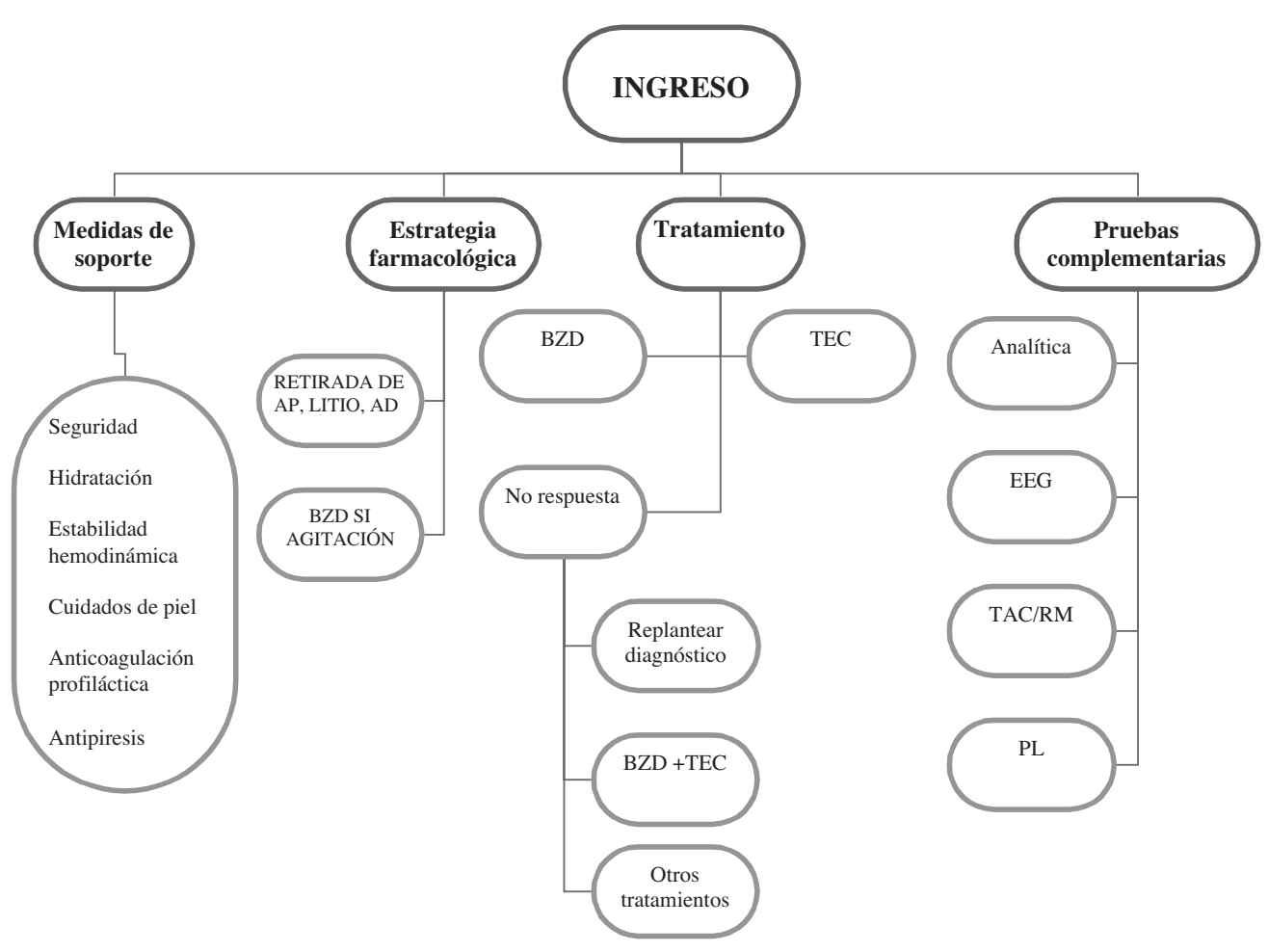




\section{BIBLIOGRAFÍA:}

(1) Kahlbaum K. Die Katatonie oder das Spannungsirresein. Eine klinische Form psychischer Krankheit. Berlin. Germany: Hirschwald, 1874.

(2) Kraepelin E. Einfiihnmg in die psychiatrische Klinik. Vol. 3. Auflage. Leipzig, Germany: Barth, 1916.

(3) Bleuler E. Dementia praecox oder Gruppe der Schizophrenien.Vol. I. Auflage. Leipzig, Germany: Deuticke, 1911.

(4) Weder ND, Muralee S, Penland H,Tampi RR. Catatonia: A Review. Ann Clin Psychiatry. 2008;20:97-107

(5) FINK M., TAYLOR MA. Catatonia: A clinician's guide to diagnosis and Treatment. Cambridge: Cambridge University Press, 2003

(6) Peralta V, Cuesta MJ. Motor features in psychotic disorders. 2. Development of diagnostic criteria for catatonia. Schizophr Res 2001; 47:117-26.

(7) Northoff G, Kock A, Wenke J, Eckert J, Boker H, Pflug B, Bogerts B. Catatonia as a psychomotor syndrome: a rating scale and extrapyramidal motor symptoms. Mov Disord 1999; 14:404-16.

(8) Arias M, Páramo M, Requena ISesar ARobelo MPeleteiro M la catatonía maligna como paradigma de enfermedad neuropsiquiátrica. Neurología 2003; 18: 107-11.

(9) Ahuja N. Organic catatonia: a review. Ind J Psychiatry 2000; 42: 327-46.

(10) AkHtar, S. Ahmad, H. Ciprofloxacin-induced catatonia. J ClinPsychiatry 1993; 54:115-6.

11.Fink M. Neuroleptic malignant syndrome and catatonia: one entity or two? Biol Psychiatry 1996; 39:1-4.

(12) CARroll BT, TAYLor BE. The nondichotomy between lethal catatonia and neuroleptic malignant syndrome. J Clin Psychopharmacol 1997; 17:235-6.

(13) FINK M. Toxic serotonin syndrome or neuroleptic malignant syndrome? Case report. Pharmacopsychiatry 1996; 29:159-61.

(14) Carroll BT, Graham KT, Thalassinos AJ: A common pathogenesis of the serotonin syndrome, catatonia, and neuroleptic malignant syndrome. J Neuropsychiatry Clin Neurosci 2001; 13:150.

(15) Fink M, TAYLOR MA, The Catatonia Syndrome. Forgotten but Not Gone. Arch Gen Psychiatry 2009; 66:1173-7.

(16) Rosebush PI, Hildebrand AM, Furlong BG, Mazurek MF. Catatonic syndrome in a general psychiatric in-patient population: frecuency, clinical presentation and reponse to lorazepam. J Clin Psychiatry $1990 ; 51: 357-62$.

(17) Van der Heijden F M, Tuinier S, Arts NJ, Hoogendoorn ML, Kahns RS, Verhoeven WM. Catatonia: disappeared or under-diagnosed? Psychopathology 2005; 38:3-8.

(18) TAYLOR MA, ABRAMS R. Catatonia: prevalence and importance in the manic phase of manicdepressive illness. Arch Gen Psychiatry 1977; 34:1223-5.

(19) American Psychiatric Association. Diagnostic and Statistical Manual of Mental Disorders, Fourth edition (DSM-IV). Arlington, VA: American Psychiatric Publishing, 1994.

(20) TAYLOR M A, FinK M. Catatonia in psychiatric classification: a home of its own. Am J Psychiatry $2003 ; 160: 1233-41$.

(21) Rosebush PI, Mazurek MF. Catatonia and its treatment. Schizophr Bull 2010; 36:239-42.

(22) Fricchione GL, CASSEm NH, Hooberman D, Hobson D: Intravenous lorazepam in neurolep- 
tic-induced catatonia. J Clin Psychopharmacol 1983; 3:338-42.

(23) TROLlor JN, SACHDEv PS: Electroconvulsive treatment of neuroleptic malignant syndrome: a review and report of cases. Aust N Z J Psychiatry 1999; 33:650-9.

(24) Bush G, Fink M, Petrides G, Dowling F, Francis A. Catatonia, II: treatment with lorazepam and electroconvulsive therapy. Acta Psychiatr Scand 1996; 93:137-43. 